\title{
On a Subclass of Close-to-Convex Functions Associated with Fixed Second Coefficient
}

\author{
Selvaraj Chellian", Stelin Simpson ${ }^{2}$, Logu Sivalingam ${ }^{1}$ \\ ${ }^{1}$ Department of Mathematics, Presidency College (Autonomous), Chennai, India \\ ${ }^{2}$ Department of Mathematics, Tagore Engineering College, Vandalur, Chennai, India
}

Email address:

pamc9439@yahoo.co.in (S. Chellian), stelinpeter@gmail.com (S. Simpson), logumaths123@gmail.com (L. Sivalingam)

\section{To cite this article:}

Selvaraj Chellian, Stelin Simpson, Logu Sivalingam. On a Subclass of Close-to-Convex Functions Associated with Fixed Second Coefficient. Applied and Computational Mathematics. Vol. 4, No. 5, 2015, pp. 342-345. doi: 10.11648/j.acm.20150405.12

\begin{abstract}
We consider a subclass of univalent functions $f(z)$ for which there corresponds a convex function $g(z)$ of order $\alpha$ such that $\operatorname{Re}\left(z f^{\prime}(z) / g(z)\right) \geq \beta$. We investigate the influence of the second coefficient of $g(z)$ on this class. We also prove distortion, covering, and radius of convexity theorems.
\end{abstract}

Keywords: Analytic Function, Univalent Function, Convex Function of Order $\alpha$, Close-to-Convexity, Fixed Second Coefficient, Radius of Convexity

\section{Introduction}

Let $\mathrm{A}$ be the class of regular functions on the unit disk $E=\{z:|z|<1\}$. Let $\mathrm{S}$ be the class of univalent functions $f(z)$ of the form

$$
f(z)=z+\sum_{n=2}^{\infty} a_{n} z^{n}
$$

normalized by the conditions $f(0)=0=f^{\prime}(0)-1$. Let $C_{b}(\alpha)$ denote the subclass of $\mathrm{S}$ consisting of functions of the form

$$
g(z)=z+\sum_{n=2}^{\infty} b_{n} z^{n}
$$

such that $\operatorname{Re}\left\{1+\frac{z g^{\prime \prime}(z)}{g^{\prime}(z)}\right\} \geq \alpha, \quad z \in E, \quad\left|b_{2}\right|=b, \quad$ and $0 \leq \alpha<1 . \mathrm{Cb}(\alpha)$ is the class of convex functions of order $\alpha$. It is known that $0 \leq \mathrm{b} \leq 1$ [2]. Moreover, $\mathrm{g}(\mathrm{z}) \in \mathrm{C} 1(\alpha)$ if and only if $g(z)=\frac{z}{1-\varepsilon z},|\varepsilon|=1$.

Definition 1.1: A normalized regular function of the form (1.1) is said to belong to the class $C_{b}^{\prime}(\alpha, \beta)$ if there exists a function $g(z) \in C_{b}(\alpha)$ of the form (1.2) such that

$$
\operatorname{Re}\left\{\frac{z f^{\prime}(z)}{g(z)}\right\} \geq \beta, \quad \beta \geq 0
$$

It is clear that for $\alpha \leq \alpha^{\prime}$ and $\beta \leq \beta^{\prime}$, we have $C_{b}^{\prime}\left(\alpha^{\prime}, \beta\right) \subset C_{b}^{\prime}(\alpha, \beta)$ and $C_{b}^{\prime}\left(\alpha, \beta^{\prime}\right) \subset C_{b}^{\prime}(\alpha, \beta)$.

Note that $C_{b}^{\prime}(0, \beta) \subset C_{b}^{\prime}(\beta)$. In [14] the author has defined a subclass $C^{\prime}$ consisting of functions $f(z)$ of the form (1.1) for which $\operatorname{Re}\left\{\frac{z f^{\prime}(z)}{g(z)}\right\}>0$ where $g(z) \in C, \mathrm{C}$ being the class of convex functions and obtained the inclusion relation $\mathrm{K}^{*} \subset \mathrm{C}^{\prime} \subset \mathrm{K} \subset \mathrm{S}^{*}, \mathrm{~K}^{*}$ is the subclass known as the class of quasi-convex functions introduced and studied by K.I. Noor [12]. Thus $C_{b}^{\prime}(\alpha, \beta) \subset C^{\prime}$ and hence every member of $C_{b}^{\prime}(\alpha, \beta)$ is univalent. By specializing $\alpha$ and $\beta$ we obtain some important subclasses. If $f(z) \in C_{b}^{\prime}(0,0)$ then $f(z)$ is close-to-convex; if $f(z) \in C_{b}^{\prime}(1, \beta)$, then $\operatorname{Re}\left\{f^{\prime}(z)\right\} \geq \beta$ that is in $\operatorname{R}(\beta)$ as in [13]; if $f(z) \in C_{b}^{\prime}(\alpha, 1)$ then $f(z)=g(z)$ so that $f(z)$ is convex of order $\alpha$. If $f(z) \in C_{b}^{\prime}(1,1)$ then $\operatorname{Re}\left\{f^{\prime}(z)\right\} \geq 0$ so $g(z)=z$.

In this paper we prove distortion, covering and radius of convexity theorems for the class $C_{b}^{\prime}(\alpha, \beta)$.

In what follows, we assume $f(z)$ is in $C_{b}^{\prime}(\alpha, \beta)$ with 
$g(z)$ its associated function in $C_{b}(\alpha)$.

First we give an example for $f(z) \in C_{b}^{\prime}(\alpha, \beta)$ by using the following.

Lemma: [16] Let $Q(z)$ be analytic for $\mathrm{z} \in \mathrm{C}$ with $Q(0)=1$. Then $\operatorname{Re} Q(z) \geq \beta$ if and only if $Q(z)=\frac{1+(1-2 \beta) G(z)}{1-G(z)}$, where $\mathrm{G}(\mathrm{z})$ is analytic,

$\mathrm{G}(0)=0$, and $|G(z)|<1$ for $z \in E$.

Example: Let

$$
\begin{gathered}
f(z)=\frac{1}{2} \int_{0}^{z} \frac{1}{t}\left[t \psi(t)+\int_{0}^{t} \psi(t) d t\right]\left(\frac{1+(1-2 \beta t)}{1-t}\right) d t \text { and } \\
g(z)=\frac{1}{2}\left[z \psi(z)+\int_{0}^{z} \psi(t) d t\right]=\frac{1}{2}\left(z \int_{0}^{z} \psi(t) d t\right) \text { where } \\
\psi(t)=\left(\frac{1}{1-t^{2}}\right)^{1-\alpha}\left(\frac{1+t}{1-t}\right)^{\frac{2 b}{3}} .
\end{gathered}
$$

Since

$$
\frac{z f^{\prime}(z)}{g(z)}=\frac{1+(1-2 \beta) z}{1-z}
$$

has real part $\geq \beta$, it suffices to show that $g(z) \in C_{b}(\alpha)$.

If $\phi(z)=\int_{0}^{z} \psi(t) d t$, then we have

$$
1+\frac{z \phi^{\prime \prime}(z)}{\phi^{\prime}(z)}=\frac{1+\frac{4 b}{3} z+(1-2 \alpha) z^{2}}{1-z^{2}}=\frac{1+(1-2 \alpha) G(z)}{1-G(z)} .
$$

Solving for $\mathrm{G}(\mathrm{z})$, we obtain

$$
G(z)=\frac{z\left\lfloor z+\frac{2 b}{3(1-\alpha)}\right\rfloor}{\left[1+\frac{2 b}{3(1-\alpha)} z\right]}=z h(z) .
$$

Since $\alpha+\mathrm{b} \leq 1, \mathrm{~h}(\mathrm{z})$ maps $\mathrm{E} \rightarrow \mathrm{E}$, and $|G(z)| \leq|z|<1$ for $\mathrm{z}$ $\in$ E. Since $G(z)$ satisfies the conditions of Lemma, $\phi(z) \in C_{b}(\alpha)$. So,

$$
g(z)=\frac{1}{2}\left[z \psi(z)+\int_{0}^{z} \psi(t) d t\right]=\frac{1}{2}\left(z \int_{0}^{z} \psi(t) d t\right)^{\prime}
$$

is convex for $|z|<\frac{1}{2}$ by Livingston [12]. Thus existence of $f(z) \in C_{b}^{\prime}(\alpha, \beta)$ is asserted.

\section{Distortion Theorem for $C_{b}^{\prime}(\alpha, \beta)$}

Theorem 2.1: Let $f(z) \in C_{b}^{\prime}(\alpha, \beta)$. Then

$$
\left|f^{\prime}(z)\right| \leq \frac{1+(1-2 \beta) r}{r(1-r)} \int_{0}^{r}\left(\frac{1}{1-t^{2}}\right)^{1-\alpha}\left(\frac{1+t}{1-t}\right)^{b} d t
$$

and

$$
\left|f^{\prime}(z)\right| \geq \frac{1-(1-2 \beta) r}{r(1+r)} \int_{0}^{r}\left(1+\frac{2 b t}{1-\alpha}+t^{2}\right)^{1-\alpha} d t
$$

where the integrand on the right hand side of (2.2) is taken to be 1 for $\alpha=1$.

Equality holds in (2.1) for the function

$$
f_{1}(z)=\int_{0}^{z} \frac{1+(1-2 \beta) s}{s(1-s)} \int_{0}^{s}\left(\frac{1}{1-t^{2}}\right)^{1-\alpha}\left(\frac{1+t}{1-t}\right)^{b} d t d s
$$

and equality holds in (2.2) for the function

$$
f_{2}(z)=\int_{0}^{z} \frac{1-(1-2 \beta) s}{s(1+s)} \int_{0}^{s}\left(1+\frac{2 b t}{1-\alpha}+t^{2}\right)^{1-\alpha} d t d s .
$$

Proof: From (1.3), by Lemma we obtain

$$
\frac{z f^{\prime}(z)}{g(z)}=\frac{1+(1-2 \beta) G(z)}{1-G(z)}
$$

where $\mathrm{G}(0)=0$ and $|G(z)|<1$ for $\mathrm{z} \in \mathrm{E}$. Since $\mathrm{G}(\mathrm{z})$ satisfies the conditions of Schwarz's lemma, (1.6) yields

$$
\frac{1-(1-2 \beta) r}{1+r} \leq\left|\frac{z f^{\prime}(z)}{g(z)}\right| \leq \frac{1+(1-2 \beta) r}{1-r}
$$

That is, $\frac{1-(1-2 \beta) r}{r(1+r)} \leq\left|\frac{f^{\prime}(z)}{g(z)}\right| \leq \frac{1+(1-2 \beta) r}{r(1-r)}$.

We have [2, p. 105],

$$
\int_{0}^{r}\left(1+\frac{2 b t}{1-\alpha}+t^{2}\right)^{\alpha-1} d t \leq|g(z)| \leq \int_{0}^{r}\left(\frac{1}{1-t^{2}}\right)^{1-\alpha}\left(\frac{1+t}{1-t}\right)^{b} d t
$$

Combining (1.7) and (1.8), the result follows. Clearly $f_{1}(z)$ and $f_{2}(z) \in C_{b}(\alpha, \beta)$ with

$$
\begin{aligned}
& g_{1}(z)=\int_{0}^{z}\left(\frac{1}{1-t^{2}}\right)^{1-\alpha}\left(\frac{1+t}{1-t}\right)^{b} d t \text { and } \\
& g_{2}(z)=\int_{0}^{z}\left(1+\frac{2 b t}{1-\alpha}+t^{2}\right)^{\alpha-1} d t .
\end{aligned}
$$

Theorem 2.2. Let $f(z) \in C_{b}^{\prime}(\alpha, \beta)$. Then

$$
\begin{gathered}
\int_{0}^{r} \frac{1-(1-2 \beta) s}{s(1-s)}\left[\int_{0}^{s}\left(1+\frac{2 b t}{1-\alpha}+t^{2}\right)^{\alpha-1} d t\right] d s \leq|f(z)| \\
\leq \int_{0}^{r} \frac{1+(1-2 \beta) s}{s(1+s)}\left[\int_{0}^{s}\left(\frac{1}{1-t^{2}}\right)^{1-\alpha}\left(\frac{1+t}{1-t}\right)^{b} d t\right] d s .
\end{gathered}
$$


Equality holds on the right-hand side for $f_{1}(z)$ in Theorem 2.1 and on the left-hand side for $f_{2}(z)$ in Theorem 2.1.

Proof: Integrating along the straight line segment from the origin to $z=r e^{i \theta}$ and applying Theorem 2.1 we obtain

$$
\begin{aligned}
|f(z)| & \leq \int_{0}^{r}\left|f^{\prime}\left(s e^{i \theta}\right)\right| d s \\
& \leq \int_{0}^{r} \frac{1+(1-2 \beta) s}{s(1+s)}\left[\int_{0}^{s}\left(\frac{1}{1-t^{2}}\right)^{1-\alpha}\left(\frac{1+t}{1-t}\right)^{b} d t\right] d s,
\end{aligned}
$$

which proves the right-hand inequality.

To prove the left-hand inequality, for every $r$ we choose $z_{0}$, $\left|z_{0}\right|=r$, such that

$$
\left|f\left(z_{0}\right)\right|=\min _{|z|=r}|f(z)|
$$

If $L\left(z_{0}\right)$ is the pre-image of segment $\left[0, f\left(z_{0}\right)\right]$, then

$$
\begin{aligned}
|f(z)| \geq\left|f\left(z_{0}\right)\right| \geq \int_{L\left(z_{0}\right)}\left|f^{\prime}(z)\right||d z| \\
\quad \geq \int_{0}^{r} \frac{1-(1-2 \beta) s}{s(1-s)}\left[\int_{0}^{s}\left(1+\frac{2 b t}{1-\alpha}+t^{2}\right)^{\alpha-1} d t\right] d s .
\end{aligned}
$$

This completes the proof.

\section{Covering Theorem for $C_{b}^{\prime}(\alpha, \beta)$}

Theorem 3.1: Let $f(z) \in C_{b}^{\prime}(\alpha, \beta)$ with

$$
\begin{gathered}
f(z)=z+\sum_{n=2}^{\infty} a_{n} z^{n} \text {. If } f(z) \neq w \text { for } \mathrm{z} \in \mathrm{E} \text {, then } \\
|w| \geq 2 /(6-2 \beta+b) .
\end{gathered}
$$

Proof: First we establish that $\left|a_{2}\right| \leq 1-\beta+\frac{b}{2}$.

Let $g(z)=z+\sum_{n=2}^{\infty} b_{n} z^{n}$. Then, for

$$
\phi(z)=\frac{1}{1-\beta}\left[\frac{z f^{\prime}(z)}{g(z)}-\beta\right]=1+\frac{\left(2 a_{2}-b_{2}\right) z}{1-\beta}+\sum_{n=2}^{\infty} c_{n} z^{n},
$$

we have [9, p. 15],

$$
\left|\frac{2 a_{2}-b_{2}}{1-\beta}\right| \leq 2, \text { so } \quad\left|a_{2}\right| \leq 1-\beta+\frac{b}{2} .
$$

Since $f(z)$ does not assume the value w,

$$
\frac{w f(z)}{w-f(z)}=z+\left(a_{2}+\frac{1}{w}\right) z^{2}+\cdots
$$

is in the class $S$. Therefore

$$
\left|a_{2}+\frac{1}{w}\right| \leq 2
$$

Now, using (3.1) and (3.2),

$$
\left|\frac{1}{w}\right|=\left|a_{2}+\frac{1}{w}-a_{2}\right| \leq 2+1-\beta+\frac{b}{2},
$$

and this completes the proof.

\section{A Radius of Convexity Theorem for $C_{b}^{\prime}(\alpha, \beta)$}

Lemma 4.1: If $f \in C_{b}^{\prime}(\beta)$, then $f(z)$ maps the disk $|z|<r_{1}$ onto a convex domain, where $\mathrm{r} 1$ is the least positive root of the equation $\gamma(r, b, \beta)=0$, where

$$
\gamma(r, b, \beta)=[1+(1-2 \beta) r](1+r)^{b}+2(1-\beta) r(1-r)^{b} .
$$

Proof: Let $f(z) \in C_{b}^{\prime}(\beta)$. Then for $g(z) \in C_{b}(0)$, $\frac{z f^{\prime}(z)}{g(z)}=Q(z)$ with $\operatorname{Re}\{\phi(z)\} \geq \beta$.

From $z f^{\prime}(z)=g(z) Q(z)$, it follows that

$$
1+\frac{\mathrm{zf}^{\prime \prime}(\mathrm{z})}{\mathrm{f}^{\prime}(\mathrm{z})}=\frac{\mathrm{zg}^{\prime}(\mathrm{z})}{\mathrm{g}(\mathrm{z})}+\frac{\mathrm{zQ} \mathrm{Q}^{\prime}(\mathrm{z})}{\mathrm{Q}(\mathrm{z})}
$$

So, the radius of convexity of $f(z)$ is at least equal to the smallest positive root of

$$
\min \operatorname{Re}\left(\frac{z g^{\prime}(z)}{g(z)}\right)+\min \operatorname{Re}\left(\frac{z Q^{\prime}(z)}{Q(z)}\right)=0
$$

For $\alpha=0$, from the inequalities in [2, p. 104] and [14, p. 384] we obtain

$$
\operatorname{Re}\left(\frac{z g^{\prime}(z)}{g(z)}\right) \geq-\left|\frac{z g^{\prime}(z)}{g(z)}\right| \geq-\frac{(1+r)^{b}}{(1-r)^{b+1}} .
$$

Now, let $Q(z)=(1-\beta) P(z)+\beta$, where $\mathrm{P}(\mathrm{z})$ is analytic, $\mathrm{P}(0)=1$, and $\operatorname{Re}\{\mathrm{P}(\mathrm{z})\}>0$ in $\mathrm{E}$.

Then $\frac{z Q^{\prime}(z)}{Q(z)}=\frac{P^{\prime}(z)}{P(z)+\frac{\beta}{(1-\beta)}}$. Using the lemma of Libera [11, p. 150] we obtain

$$
\left|\frac{z Q^{\prime}(z)}{Q(z)}\right| \leq \frac{2(1-\beta) r}{(1-r)[1+(1-2 \beta) r]} .
$$

Using (4.2) and (4.3) in (4.1) we get 


$$
\begin{aligned}
\operatorname{Re}\left(1+\frac{z f^{\prime \prime}(z)}{f^{\prime}(z)}\right) & \geq-\frac{(1+r)^{b}}{(1-r)^{b+1}}-\frac{2(1-\beta) r}{(1-r)[1+(1-2 \beta) r]} \\
& \geq-\frac{\gamma(r, b, \beta)}{(1-r)^{b+1}[1+(1-2 \beta) r]}
\end{aligned}
$$

Hence, $f(z)$ is convex in $|z|<r_{1}$ where $\mathrm{r} 1$ is the least positive root of the equation $\gamma(r, b, \beta)=0$ for given $b, \beta$. This lemma improves the result obtained in [16].

Theorem 4.2: If $f(z) \in C_{b}^{\prime}(\alpha, \beta)$, then $f(z)$ maps the disk $|z|<R$ onto a convex domain, where $\mathrm{R}$ is the least positive root of the equation $\delta(r, b, \alpha, \beta)=0$, where

$$
\begin{aligned}
\delta(r, b, \alpha, \beta) & =(1-2 \alpha)(1-2 \beta) r(1+r)^{b+\alpha-1}(1-r)^{\alpha-b} \\
& +(1-2 \alpha)(1+r)^{b+\alpha-1}(1-r)^{\alpha-b} \\
& -2(1-\beta)(1+r)^{2 \alpha-1}+2(1-\beta) .
\end{aligned}
$$

Proof: Let $f(z) \in C_{b}^{\prime}(\alpha, \beta)$. Then for $\frac{z f^{\prime}(z)}{g(z)}=Q(z)$ with $\operatorname{Re}\{\phi(z)\} \geq \beta$.

From $z f^{\prime}(z)=g(z) Q(z)$, it follows that

$$
1+\frac{\mathrm{zf}^{\prime \prime}(\mathrm{z})}{\mathrm{f}^{\prime}(\mathrm{z})}=\frac{\mathrm{zg}(\mathrm{z})}{\mathrm{g}(\mathrm{z})}+\frac{\mathrm{zQ}(\mathrm{z})}{\mathrm{Q}(\mathrm{z})}
$$

So, the radius of convexity of $f(z)$ is at least equal to the smallest positive root of

$$
\min \operatorname{Re}\left(\frac{z^{\prime}(z)}{g(z)}\right)+\min \operatorname{Re}\left(\frac{z Q^{\prime}(z)}{Q(z)}\right)=0 .
$$

Using the inequalities in [2, p. 104] and [14, p. 384] we obtain

$$
\begin{aligned}
\operatorname{Re}\left(\frac{z g^{\prime}(z)}{g(z)}\right) & \geq-\left|\frac{z g^{\prime}(z)}{g(z)}\right| \\
& \geq-\frac{(2 \alpha-1) r(1+r)^{b}}{\left(1-r^{2}\right)^{1-\alpha}(1-r)^{b}\left[(1+r)^{2 \alpha-1}-1\right]}
\end{aligned}
$$

Now, let $Q(z)=(1-\beta) P(z)+\beta$, where $\mathrm{P}(\mathrm{z})$ is analytic, $\mathrm{P}(0)=1$, and $\operatorname{Re}\{\mathrm{P}(\mathrm{z})\}>0$ in $\mathrm{E}$.

Then $\frac{z Q^{\prime}(z)}{Q(z)}=\frac{P^{\prime}(z)}{P(z)+\frac{\beta}{(1-\beta)}}$. Using the lemma of Libera [11, p. 150] we obtain

$$
\left|\frac{z Q^{\prime}(z)}{Q(z)}\right| \leq \frac{2(1-\beta) r}{(1-r)[1+(1-2 \beta) r]}
$$

Using (4.5) and (4.6) in (4.4) we get

$$
\begin{aligned}
& \operatorname{Re}\left(1+\frac{z f^{\prime \prime}(z)}{f^{\prime}(z)}\right) \geq-\frac{(2 \alpha-1) r(1+r)^{b}}{\left(1-r^{2}\right)^{1-\alpha}(1-r)^{b}\left[(1+r)^{2 \alpha-1}-1\right]} \\
&-\frac{2(1-\beta) r}{(1-r)[1+(1-2 \beta) r]} \\
& \geq-\frac{r \delta(r, b, \alpha, \beta)}{(1-r)\left[(1+r)^{2 \alpha-1}\right][1+(1-2 \beta) r]} .
\end{aligned}
$$

Hence, $f(z)$ is convex in $|z|<R$ where $\mathrm{R}$ is the least positive root of the equation $\delta(r, b, \alpha, \beta)=0$, for given $\mathrm{b}, \alpha$, $\beta$.

\section{References}

[1] O.P. Ahuja, "The influence of second coefficient on spirallike and Robertson functions", Yokohama Math. J. 34(1-2) (1986) $3-1$.

[2] H.S. Al-Amiri, "On close-to-star functions of order $\alpha$ ", Proc. Amer. Math. Soc. 29 (1971) $103-108$.

[3] V.V. Anh, "Starlike functions with a fixed coefficient", Bult. Austral. Math. Soc. 39(1) (1989) $145-158$.

[4] P.L. Duren, "Univalent functions", Springer-Verlag, N.Y. Berlin, Heidelberg, Tokyo, 1983.

[5] M. Finkelstein, "Growth estimates of convex functions", Proc. Amer. Math. Soc. 18 (1967), $412-418$.

[6] R.M. Goel, "The radius of convexity and starlikeness for certain classes of analytic functions with fixed coefficient", Ann. Univ. Mariar Euric Sklodowska Sect. A, 25 (1971) 33 39.

[7] A.W. Goodman, "Univalent functions", Vol. I, II, Mariner Tampa, Florida, 1983.

[8] T.H. Gronwall, "On the distortion in conformal mapping when the second coefficient in the mapping function has an assigned value”, Prof. Nat. Acad. Proc. 6 (1920) $300-302$.

[9] W.K. Hayman, "Multivalent functions", Cambridge University Press, 1958.

[10] W. Kaplan, "Close-to-convex functions", Mich. Math. J. 1 (1952) $169-185$.

[11] R.J. Libera, "Some radius of convexity problems", Duke Math. J. 31 (1964) $143-158$.

[12] A.E. Livingston, "On the radius of univalence of certain analytic functions", Proc. Amer. Math. Soc. 17 (1965) 352 357.

[13] K.I. Noor, "Radius problem for a subclass of close-to-convex univalent functions", Int. J. Math. Sci. 14(4) (1992) $719-726$.

[14] M.S. Robertson, "On the theory of univalent functions", Ann. Math. 37 (1936) $374-408$.

[15] C. Selvaraj, "A subclass of close-to-convex functions", Southeast Asian Bull. Math., 28 (2004) 113 - 123.

[16] C. Selvaraj and N. Vasanthi, "A certain subclass of close-toconvex functions defined in the unit disk", Far East J. Math. Sci. 24(2) (2010) $241-253$.

[17] H. Silverman, "On a close-to-convex functions", Proc. Amer. Math. Soc. 36(2) (1972) $477-484$. 